Universidade de São Paulo

Escola Superior de Agricultura "Luiz de Queiroz"

Voláteis de flores de café com etanol e metanol são sinérgicos para a atração de Hypothenemus hampei (Ferrari) em cafezais

Mariana Oliveira Garrigós Leite

Dissertação apresentada para obtenção do título de Mestra em Ciências. Área de concentração:

Entomologia

Piracicaba

2016 


\section{RESUMO \\ Voláteis de flores de café com etanol e metanol são sinérgicos para a atração de Hypothenemus hampei (Ferrari) em cafezais}

Neste estudo, foi testada a atratividade de novos semioquímicos a fêmeas da broca-do-café, Hypothenemus hampei (Ferrari), que é a principal praga da cultura do café no mundo. Armadilhas iscadas com Trat. 1, Trat. 2 e Trat. 3 foram testadas em combinação ou não com etanol e metanol 'E:M' (mistura 1:1) em uma área de cafezal localizada em Inconfidentes-MG, Brasil. O resultado mais importante foi que armadilhas iscadas com Trat. 1 + E:M atraíram significantemente mais adultos da broca-do-café do que os outros tratamentos isolados ou combinados. Por outro lado, armadilhas iscadas com compostos voláteis de frutos de café Trat. 2 + E:M não diferiram de E:M. Já armadilhas iscadas com Trat. 3 + E:M atraíram menos besouros do que E:M. Um número significativo de outras espécies de Scolytinae também foram atraídos por armadilhas iscadas com Trat. 1 + E:M e E:M. Além disso, um número muito baixo de outros insetos de, pelo menos, oito ordens também foram atraídos por estes dois tratamentos. Tomados juntos, estes resultados oferecem evidências convincentes de que Trat. 1 e E:M são sinérgicos para a captura de $H$. hampei, favorecendo o desenvolvimento de uma nova estratégia para o manejo integrado desta espécie em cafezais.

Palavras-chave: Ecologia química; voláteis de plantas; armadilhas; insetos não-alvo; manejo integrado de pragas 


\section{ABSTRACT \\ Coffee flower volatiles with ethanol and methanol are synergistic for the attraction of Hypothenemus hampei (Ferrari) in coffee growing areas}

In this study, it was tested the attractiveness of new semiochemicals to coffee berry borer females, Hypothenemus hampei (Ferrari), that is the most important pest of coffee in the world. Traps baited with Treat. 1, Treat. 2 and Treat. 3 were tested in combination or not with ethanol and methanol "E:M" (1:1 mixture) in a coffee growing area located in Inconfidentes, MG, Brazil. The most relevant result is that traps baited with Treat. 1 + E:M attracted significantly more coffee berry borer adults than the other treatments. On the other hand, traps baited with coffee fruits volatile compounds Treat. $2+\mathrm{E}$ :M were similar to the ones bated with E:M. Yet traps with Treat. $3+$ E:M attracted significantly less beetles than traps with E:M. A significant number of other Scolytinae species were also attracted by Treat. $1+\mathrm{E}: \mathrm{M}$ and E:M. Moreover, a very low number of other insects from, at least, eight orders were also attracted by these two treatments. Taken together, these results offer compelling evidences that Treat. 1 and E:M are synergistic for the capture of $H$. hampei, promoting the development of a new strategy for the integrated management of this species in coffee growers.

Keywords: Chemical ecology; Plant volatiles; Traps; Non-target insects; Integrated pest management 


\section{INTRODUÇÃO}

A broca do café, Hypothenemus hampei (Ferrari) (Coleoptera: Curculionidae: Scolytinae) é a principal praga da cafeicultura no mundo, causando perdas quantitativas e qualitativas na produção de café (INFANTE; PÉREZ; VEGA, 2012). Somente no Brasil, os prejuízos anuais por esta praga ultrapassam 300 milhões de dólares (OLIVEIRA et al., 2013).

A broca-do-café possui comportamento críptico, passando todo o seu ciclo de vida (ovo a adulto) dentro do fruto de café (BERGAMIN, 1943), o que dificulta a ação dos agentes de manejo da praga (DAMON, 2000). As poucas estratégias disponíveis para manejo de $H$. hampei têm sido baseadas, principalmente, no uso de inseticidas sintéticos, tais como aqueles do grupo do endosulfan (DAMON, 2000). Contudo, devido a toxicidade ao homem e poluição do meio ambiente, além do surgimento de casos de resistência de pragas relacionadas a estes produtos (BRUN et al., 1989, BRUN; MARCILLAUD; GAUDICHON, 1994, DAMON, 2000), seu uso foi banido em diversas partes do mundo. No Brasil, o uso de endosulfan foi proibido a partir de 2013, mas, desde então, surtos populacionais de $H$. hampei têm sido relatados nas principais regiões produtoras do país (PEREIRA et al., 2012). Por essa razão, a busca por novas estratégias para o manejo da broca-do-café, tais como o uso de semioquímicos tem se tornado cada vez mais importantes (JARAMILLO; BORGEMEISTER; BAKER, 2006, ARISTIZÁBAL; BUSTILLO; ARTHURS, 2016).

Após a cópula, fêmeas de $H$. hampei normalmente abandonam o fruto de café no qual se desenvolveram, visando a colonização de novos frutos. Por esta razão são chamadas de 'fêmeas colonizadoras' (MATHIEU et al. 1997, 2001). Fatores ambientais como temperatura, umidade (BAKER; BARRERA; RIVAS, 1992) e luz (MATHIEU et al. 1997), assim como o estado da musculatura alar (LÓPEZ-GUILLÉN et al., 2011), e do tegumento do inseto (SILVA et al., 2014), são primordiais para desencadear o processo de dispersão da espécie.

No campo, aparentemente, fêmeas colonizadoras de $H$. hampei utilizam os voláteis dos frutos de café para localizar um novo hospedeiro (GIORDANENGO; BRUN; FRÉROT, 1993). Neste caso, a intensidade da resposta olfativa do inseto varia com o estágio de amadurecimento do fruto, sendo preferidos os maduros ao invés dos verdes ou secos (GIORDANENGO; BRUN; FRÉROT, 1993). A presença de álcoois nos voláteis destes frutos parece ser uma das explicações pelas quais armadilhas 
contendo etanol e metanol capturam a broca-de-café (MENDOZA-MORA, 1991). De fato, armadilhas contendo a mistura 1:1 ou 1:3 de etanol e metanol têm sido amplamente utilizadas para monitoramento e manejo populacional de $H$. hampei (DUFOUR; FRÉROT, 2008, FERNANDES et al., 2011, PEREIRA et al., 2012). Estudos mais detalhados têm demonstrado respostas eletrofisiológicas em antenas de fêmeas colonizadoras a alguns compostos presentes nos voláteis de frutos de café (MENDESIL et al., 2009, JARAMILLO et al., 2013; ROBLERO; MALO, 2013). Por outro lado, a incorporação destes voláteis em armadilhas não tem resultado em aumentos de capturas da broca-do-café em comparação as obtidas com etanol e metanol (NJIHIA et al., 2014). Recentemente, novos compostos eletrofisiologicamente ativos a H. hampei foram identificados nos voláteis de flores de café (Silva, 2014), porém sua resposta no campo ainda não foi investigada.

Tomadas juntas, estas informações demostram muitas lacunas acerca da utilização de semioquímicos no manejo de $H$. hampei. Neste sentido, este trabalho teve por objetivo, selecionar a melhor composição química para a captura da broca-do-café, $H$. hampei, a partir de compostos voláteis eletrofisiologicamente ativos, isoladamente, ou em mistura com outros compostos, já utilizados na comunicação química de diferentes espécies de Scolytinae. Adicionalmente, considerando que muitas espécies não alvo, como outros escolitíneos, também são capturadas nestas armadilhas, este trabalho visou ainda, avaliar o impacto destes atraentes na captura destes insetos. 


\section{CONCLUSÕES}

Com base nos resultados obtidos no presente trabalho, pode-se concluir que:

- Compostos voláteis EAD-ativos do Trat. 1 em combinação com etanol e metanol (E:M) promovem capturas de adultos de H. hampei superiores as obtidas com apenas E:M, indicando um efeito sinergético entre Trat. 1 e E:M;

- Armadilhas iscadas com alguns dos compostos EAD-ativos encontrados em Trat. 2 em combinação ou não com E:M resultam em capturas iguais ou inferiores as obtidas com E:M;

- Armadilhas iscadas com alguns dos compostos voláteis encontrados em Trat. 3 possui efeito antagônico sobre E:M;

- Armadilhas iscadas com Trat. 1 + E:M, assim como E:M capturam uma grande quantidade de outras espécies de Scolytinae;

- Armadilhas iscadas com Trat. 1 + E:M, assim como E:M capturam menos insetos de outras espécies não alvos, em relação a $H$. hampei e outros escolitíneos, indicando um baixo impacto destes atraentes sobre potenciais inimigos naturais;

- Armadilhas iscadas com Trat. 1 + E:M possuem potencial de serem empregadas no manejo integrado da broca-do-café. 


\section{REFERÊNCIAS}

AGÊNCIA NACIONAL DE VIGILÂNCIA SANITÁRIA. Disponível em:

<http://portal.anvisa.gov.br/wps/portal/anvisa/home >. Acesso em: 29 mar 2015.

ALVIM, P.T. Moisture stress as a requirement for flowering of coffee. Science, Washington, v. 132, p. 354, 1960.

ARISTIZÁBAL, L.F.; BUSTILLO, A.E.; ARTHURS, S.P. Integrated Pest Management of Coffee Berry Borer: Strategies from Latin America that Could Be Useful for Coffee Farmers in Hawaii. Insects, Basel, v. 7, p. 1-24, 2016.

ATKINS, M.D. Behavioral variation among scolytids in relation to their habitat. The Canadian Entomologist, Alberta, v. 98, p. 285-288, 1966.

BAKER, P.S. Some aspects of the behaviour of the coffee berry borer in relation to its control in southern Mexico (Coleoptera: Scolytidae). Folia Entomológica Mexicana, Montecillo, v. 61, p. 9-24, 1984.

BAKER, P.S. The coffee berry borer in Colombia. Final report of the DFID- CenicaféCABI Bioscience IPM for coffee project. Chinchiná. (Colombia), DFID - CENICAFÉ, 1999. 154p.

BAKER, P.S.; BARRERA, J.F.; RIVAS, A. Life-History studies of the coffee berry borer (Hypothenemus hampei, Scolytidae) on coffee trees in southern Mexico. The Journal of Applied Ecology, Oxford, UK, v. 29, n. 3, p. 656-662, 1992.

BARRERA, J.F. Investigación sobre la broca del café en México: logros, retos y perspectivas. In: SIMPOSIO SOBRE LA SITUACIÓN ACTUAL Y PERSPECTIVAS DE LA INVESTIGACIÓN Y MANEJO DE LA BROCA DEL CAFÉ EN COSTA RICA, CUBA, GUATEMALA Y MÉXICO, 2005, Tapachula. Anais... Tapachula: Sociedad Mexicana de Entomología, 2005.p. 1-13.

BARRERA, J. F.; HERRERA, J.; CHIU, M.; GÓMEZ, J.; VALLE-MORA, J. La trampa de una ventana (ECOIAPAR) captura más broca del café Hypothenemus hampei que la trampa de tres ventanas (ETOTRAP). Entomologia Mexicana, Montecillo, v. 7, p. 619-624, 2008.

BENASSI, V.L.R.M. Levantamento dos inimigos naturais da broca-do-café, Hypothenemus hampei (Ferr.) (Coleoptera: Scolytidae) no norte do Espírito Santo.

Anais da Sociedade Entomológica do Brasil, Londrina, v. 24, n. 3, p. 635-638, 1995. 
BENASSI, V.L.R.M. Parasitóides da broca-do-café no Brasil: histórico e perspectivas. In: MANEJO DA BROCA-DO-CAFÉ, 2007, Londrina. Anais do manejo da brocado-café Workshop Internacional. Londrina: Instituto Agronômico do Paraná, 2007. p. 193-198.

BERGAMIN J. Contribuição para o conhecimento da biologia da broca-do-café Hypothenemus hampei (Ferrari, 1867) (Coleoptera: Ipidae). Arquivos do Instituto Biológico, São Paulo, v. 14, p.31-72, 1943.

BERTHET, J.J.A. Caruncho do café: informação prestada pelo Snr. Director do Instituto Agronomico a respeito de amostras de café vinda do Congo Belga. Boletim de Agricultura, São Paulo, v. 14, p. 312-313, 1913.

BORBÓN-MARTÍNEZ, O.; O. MORA-ALFARO, A.; CAM-OEHLSCHLAGER, L. M. GONZÁLEZ. Proyecto de trampas, atrayentes y repelentes para el control de la broca del fruto de cafeto, Hypothenemus hampei L. (Coleoptera: Scolytidae). In: SIMPOSIO LATINOAMERICANO DE CAFICULTURA, 19., 2000, San José. Annais... San José: IICA, 2000. p. 331-348.

BRUN, L.O.; MARCILLAUD, C.; GAUDICHON, V.; SUCKLING, D.M. Endosulfan Resistance in Hypothenemus hampei (Coleoptera: Scolytidae) in New Caledonia. Journal of Economic Entomology, Lanham, v. 82, p. 1311-1316, 1989.

BRUN, L.O.; MARCILLAUD, C.; GAUDICHON, V.; SUCKLING, D.M. Cross resistance between insecticides in coffee berry borer, Hypothenemus hampei (Coleoptera: Scolytidae) from New Caledonia. Bulletim of Entomological Research, Cambridge, v. 84, p. 175-178, 1994.

BUSTILLO, A.E. Desarrollo de un programa de manejo integrado de la broca del café: Innovación y Ciencia, Bogotá, v. 5, p. 23-38, 1998.

BUSTILLO, A.E.; CÁRDENAS, R.; POSADA, F.J. Natural enemies and competitors of Hypothenemus hampei (Ferrari) (Coleoptera: Scolytidae) in Colombia. Neotropical Entomology, Londrina, v. 31, n. 4, p. 635-639, 2002.

BYERS, J.A. Pheromone biosynthesis in the bark beetle, Ips paraconfusus, during feeding or exposure ro vapours of host plant precursors. Insect Biochemistry, London , v.11, p. 563-569, 1981. 
BYERS, J.A.; LANNE, B.S.; LÖFQVIST, J. Host-tree unsuitability recognized by pine shoot beetles in flight. Experientia, Basel, v. 45, p. 489-492, 1989.

BYERS, J.A.; ZHANG, Q.H.; BIRGERSSON, G. Avoidance of nonhost plants by a bark beetle, Pityogenes bidentatus, in a forest of odors. Naturwissenschaften, New York, v. 91, p. 215-219, 2004.

BYERS, J.A.; ZHANG, Q.H.; SCHLYTER, F.; BIRGERSSON, G. Volatiles from nonhost birch trees inhibit pheromone response in spruce bark beetles.

Naturwissenschaften, New York, v. 85, p. 557-561, 1998.

CABI. Invasive Species Compendium. Disponível em:

$<$ http://www.cabi.org/isc/?compid=5\&dsid=51521\&loadmodule=datasheet\&page $=481$ \&site=144>. Acesso em: 15 mar. 2016.

CAMILO, J.E.; OLIVARES, F.F.; JIMÉNEZ, H.A. Fenología y reproducción de la broca del café (Hypothenemus hampei Ferrari) durante el desarrollo del fruto.

Agronomía Mesoamericana, Alajuela, v. 14, n. 1, p. 59-63, 2003.

CAMPOS-ALMENGOR, O.G. Estudio de hábitos de la broca del fruto del café (Hypothenemus hampei, Ferr. 1867) en el campo. In: SIMPOSIO LATINO-

AMERICANO SOBRE CAFICULTURA, SAN SALVADOR, 10., 1982. San Salvador.

Ponencias, Resultados y Recomendaciones de Eventos Técnicos No. 323, San

Salvador: Instituto Interamericano de Cooperación para la Agricultura, 1982. p. 38-49.

CÁRDENAS, R. Trampas y atrayentes para monitoreo de poblaciones de la broca del café, Hypothenemus hampei (Ferrari) (Col., Scolytidae), In: SIMPOSIO

LATINOAMERICANO DE CAFICULTURA, 19., Costa Rica, 2000. Anais... San José, Costa Rica, 2.000. p. 369- 379.

CEJA-NAVARRO, J.A.; VEGA, F.E,; KARAOZ, U.; HAO, Z.; JENKINS, S.; LIM, H.C.; KOSINA, P.; INFANTE, F.; NORTHEN, T.R.; BRODIE, E.L. Gut microbiota mediate caffeine detoxification in the primary insect pest of coffee. Nature

Communications, London, v. 6, p. 7618, 2015.

COMPANHIA NACIONAL DE ABASTECIMENTO. Conjuntura Semanal.

Disponível em:

<http://www.conab.gov.br/OlalaCMS/uploads/arquivos/16_01_20_17_01_56_boletim_ cafe_-_janeiro_2016.pdf $\geq$. Acesso em: 25 abril 2016. 
CORBETT, G.H. Some Preliminary Observations on the Coffee Berry Beetle Borer Stephanoderes (Cryphalus) hampei Ferr. Malayan Agricultural Journal, Kuala Lumpur, v. 21, n. 1, p. 8-22, 1933.

COSTA, G.F.; FARIA, C.A. ¿Por qué fêmeas da broca do café perfuram preferencialmente a coroa dos frutos?. Academia Insecta, Viçosa, v. 1, n. 1, p. 1-4, 2001.

CRUZ-LÓPEZ, L.; DÍAZ-DÍAZ, B.; ROJAS, J.C. Coffee volatiles induced after mechanical injury and beetle herbivory attract the coffee berry borer and two of its parasitoids. Arthropod-Plant Interactions, Dordrecht, v. 10, p. 151-159, 2016.

DAMON, A. A review of the biology and control of the coffee berry borer, Hypothenemus hampei (Coleoptera: Scolytidae). Bulletin of Entomological Research, London, v. 90, p. 453-465, 2000.

DAVIS; A.P.; GOVAERTS, R.; BRIDSON, D.M.; STOFFELEN, P. An annotated taxonomic conspectus of the genus Coffea (Rubiaceae). Botanical Journal of the Linnean Society, London, v. 152, p. 465-512, 2006.

DUFOUR, B.; FRÉROT, B. Optimization of coffee berry borer, Hypothenemus hampei Ferrari (Col., Scolytidae), mass trapping with an attractant mixture. Journal of Applied Entomology, Berlin, v. 132, p. 591-600, 2008.

EMURA, M.; NOHARA, I.; TOYODA, T.; KANISAWA, T. The volatile constituents of the coffee flower (Coffea arabica L.). Flavour and fragrance journal, Hoboken, v. 12, p. 9-13, 1997.

ERBILGIN, N.; KROKENE, P.; KVAMME, T.; CHRISTIANSEN, E. A host monoterpene influences Ips typographus (Coleoptera: Curculionidae, Scolytinae) responses to its aggregation pheromone. Agricultural and Forest Entomology, Malden, v. 9, p. 35-140, 2007.

ERBILGIN, N.; RAFFA, K.F. Opposing effects of host monoterpenes on responses by two sympatric species of bark beetles to their aggregation pheromones. Journal of Chemical Ecology, Dordrecht, v. 26, n. 11, p. 2527-2548, 2000.

FERNANDES, F.L.; PICANÇO, M.C.; CAMPOS, S.O.; BASTOS, C.S.; CHEDIAK, M.; GUEDES, R.N.C.; da SILVA, R.S. Economic Injury Level for the Coffee Berry Borer (Coleoptera: Curculionidae: Scolytinae) Using Attractive Traps in Brazilian Coffee Fields. Journal of Economic Entomology, Lanham, v. 104, p. 19091917, 2012. 
FERNANDEZ, F.; SHARKEY, M.J. Introduccion a los Hymenoptera de la región neotropical. Bogotá: Sociedad Colombiana de Entomologia y universidad Nacional de Colombia, 2006. p. 521-538.

FERNANDEZ, S.; CORDERO, J. Biología de la broca del café Hypothenemus hampei (Ferrari) (Coleoptera: Curculionidae: Scolytinae) en condiciones de laboratorio.

Bioagro, Barquisimeto, v. 19, n. 1, p. 35 - 40, 2007.

FETTIG, C.J.; MCKELVEY, S.R.; DABNEY, C.P.; HUBER, D.P.W. Responses of Dendroctonus brevicomis (Coleoptera: Curculionidae) in behavioral assays: implications to development of a semiochemical-based tool for tree protection. Forest Entomology, Ottawa v. 105, n. 1, p. 149-160, 2012.

GIORDANENGO, P.; BRUN, L.O.; FRÉROT, B. Evidence for allelochemical attraction of the coffee berry borer, Hypothenemus hampei, by coffee berries. Journal of Chemical Ecology, Dordrecht, v. 19, p. 763-769, 1993.

GITAU, C.W.; BASHFORD, R.; CARNEGIE, A.J.; GURR, G.M. A review of semiochemicals associated with bark beetle (Coleoptera: Curculionidae: Scolytinae) pests of coniferous trees: A focus on beetle interactions with other pests and their associates. Forest Ecology and Management, Amsterdam, v. 297, p. 1-14, 2013.

GONTHIER, D.J.; ENNIS, K.K.; PHILPOTT, S.M.; VANDERMEER, J.; PERFECTO, I. Ants defend coffee from berry borer colonization. BioControl, Dordrecht, v. 58, p. 815-820, 2013.

GONZÁ LEZ, M.O.; DUFOUR, B.P. Diseño, desarrollo y evaluación de trampas para el manejo integrado de la broca del café Hypothenemus hampei Ferr. en El Salvador.

Boletín Promecafe, São Salvador, v. 91/92, p. 11-19, 2001.

GUTIERREZ-MARTÍNEZ, A.; HERNÁNDEZ-RIVAS, S.; VIRGEN, A. Trampeo en el campo de la broca del fruto del café Hypothenemus hampei Ferrari (Coleoptera: Scolytidae) con los semioquímicos volátiles del fruto de café (Coffea canephora) Pierre ex Froehner, In: SIMPOSIO LATINOAMERICANO DE CAFICULTURA, 16., 1995, Managua. Anais... Managua: IICA, 1995, p. 51-52.

GUTIÉRREZ-MARTÍNEZ, A.; ONDARZA, R.N. Kairomone effect of extracts from Coffea canephora over Hypothenemus hampei (Coleoptera: Scolytidae).

Environmental Entomology, Lanham, v. 25, p. 96-100, 1996. 
GUTIÉRREZ-MARTÍNEZ, A.; RIVAS, S.H.; SANCHEZ, A.V. Atracción química de la broca del fruto de café Hypothenemus hampei Ferrai (Coleoptera: Scolytidae) por las diferentes variedades de café en el Sononusco, Chiapas, México. In: SIMPOSIO LATINOAMERICANO DE CAFEICULTURA, 1993, Managua. Anais..., Manágua: IICA, 1993. v. 16, p.1-8.

HUBER, D.P.W.; GRIES, R.; BORDEN, J.H. PIERCE Jr., H.D. A survey of antennal responses by five species of coniferophagous bark beetles (Coleoptera: Scolytidae) to bark volatiles of six species of angiosperm trees. Chemoecology, Basel, v. 10, p. 103113, 2000.

INAFNTE, F.; MUMFORD, J.; BAKER, P. Life history studies of Prorops nasuta, a parasitoid of the coffee berry borer. BioControl, Dordrecht, v 50, p. 259-270, 2005.

INFANTE, F., PÉREZ, J.; VEGA, F.E. Redirect research to control coffee pest. Nature, London, v. 489, p. 502, 2012.

JARAMILLO, J.; BORGEMEISTER, C.; BAKER, P. Coffee berry borer Hypothenemus hampei (Coleoptera: Curculionidae): searching for sustainable control strategies. Bulletin of Entomological Research, Cambridge, v. 96, p. 223-233, 2006.

JARAMILLO, J.; CHABI-OLAYE, A; POEHLING, H.; KAMONJO, C.; BORGEMEISTER, C. Development of an improved laboratory production technique for the coffee berry borer Hypothenemus hampei, using fresh coffee berries.

Entomologia Experimentalis et Applicata, Dordrecht, v. 130, p. 275-281, 2009.

JARAMILLO, J.; TORTO, B.; MWENDA, D.; TROEGER, A.; BORGEMEISTER, C.; POEHLING, H.; FRANCKE, W. Coffee Berry Borer Joins Bark Beetles in Coffee Klatch, PlosOne, São Francisco, v. 8, p. 1-15, 2013.

LACEY, E.S.; MOREIRA, J.A.; MILLAR, J.G.; HANKS, L.M. A male-produced aggregation pheromone blend consisting of alkanediols, terpenoids, and an aromatic alcohol from the cerambycid beetle Megacyllene caryae. Journal of Chemical Ecology, Dordrecht, v. 34, p. 408-417, 2008.

LAUZIÈRE, I.; BRODEUR, J.; PÉREZ-LACHAUD, G. Host stage selection and suitability in Cephalonomia stephanoderis Betrem (Hymenoptera: Bethylidae), a parasitoid of the coffee berry borer, Biological Control, Orlando, v. 21, p. 128-133, 2001. 
LEROY, J.V. Obervations relatives à quelques insectes attaquant le Caféier.

Publications de l'Institut National pour l'É tude Agronomique du Congo Belge, Série

Scientifique, Bruxelas, v. 8, p. 30, 1936.

LINGENHELD, M. Coffee's Weakness Masks Bullish Fundamentals, Forbes, New York, p.1-4, 2016.

LIU, Y.B.; MCLEAN, J.A. Field evaluation of responses of Gnathotrichus sulcatus and G. retusus (Coleoptera: Scolytidae) to semiochemicals. Journal of Economic Entomology, Lanham, v. 82, p. 1688-1690, 1989.

LIU, Y.; DAI, H. Application of bark beetle semiochemicals for quarantine of bark beetles in China. Journal of Insect Science, Oxford, v. 6, p. 1-6, 2006.

LÓPEZ-GUILLÉN, G.; VALDEZ-CARRASCO, J.; CRUZ-LÓPEZ, L.; BARRERA, J.F.; MALO, E.A.; ROJAS, J.C. Morphology and structural changes in flight muscles of Hypothenemus hampei (Coleoptera: Curculionidae) females. Environmental Entomology, Lanham, v. 40, p. 441-448, 2011.

MATHIEU, F.; BRUN, L. O.; MARCHILLAUD, C.; FRÉROT, B. Trapping of the coffee berry borer Hypothenemus hampei Ferr. (Col., Scolytidae) within a meshenclosed environment: interaction of olfactory and visual stimuli. Journal of Applied Entomology, Berlin, v. 121, p. 181-186, 1997.

MATHIEU, F.; GAUDICHON, V.; BRUN, L.O.; FRÉROT, B. Effect of physiological status on olfactory and visual responses of female Hypothenemus hampei during host plant colonization. Physiological Entomology, Oxford, v. 26, p.189- 193, 2001.

MATHIEU, F.; MALOSSE, C.; FRÉROT, B. Identification of the volatile components released by fresh coffee berries at different stages of ripeness. Journal of Agriculture and Food Chemistry, Easton, v. 46, p. 1106-1110, 1998.

MENDESIL, E.; BRUCE, T.J.A.; WOODCOCK, C.M.; CAULFIELD, J.C.; SEYOUM, E.; PICKETT, J.A. Semiochemicals used in host location by the coffee berry borer, Hypothenemus hampei. Journal of Chemical Ecology, Dordrecht, v. 35, p. 944-950, 2009.

MENDESIL, E.; JEMBERE, B.; SEYOUM, E. Population dynamics and distribution of the coffee berry borer, Hypothenemus hampei (Ferrari) (Coleoptera: Scolytidae) on Coffea arabica L. in southwestern Ethiopia. Sinet, Addis Ababa, v. 27, p. 127-134, 2004. 
MENDOZA-MOORA, J.R. Resposta da broca-do-café, Hypothenemus hampei, a estímulos visuais e semioquímicos, 1991. 44p. Tese (Entomologia na área de ) Universidade Federal de Viçosa, Minas Gerais, Brasil, 1991.

MESSING, R.H. The coffee berry borer (Hypothenemus hampei) invades Hawaii: Preliminary investigations on trap response and alternate hosts. Insects, Basel, v. 3, p. 640-652, 2012.

MOECK, H.A. Ethanol induces attack on trees by spruce beetles, Dendroctonus rufipennis (coleoptera: scolytidae). The Canadian Entomologist, Alberta, v. 113, p. 939-942, 1981.

MORALLO-REJESUS, B.; BALDOS, E.P.; TEJADA, A.M. Evaluation of insecticides against coffee berry borer and its residues in processed coffee. Philippines

Entomology, Laguna, v. 4, p. 415-433, 1980.

MORRIS, J.R.; VANDERMEER, J.; PERFECTO, I. A Keystone Ant Species Provides Robust Biological Control of the Coffee Berry Borer Under Varying Pest Densities. PLoS ONE, São Francisco, v. 10, p. 1-15, 2015.

MULLER, K.E.; FETTERMAN, B.A. Regression and ANOVA: An Integrated Approach using SAS ${ }^{\circledR}$ Software. SAS Institute: Cary, NC, 2002.

MURPHY, S.T.; MOORE, D. Biological control of the coffee berry borer, Hypothenemus hampei (Ferrari) (Coleoptera: Scolytidae): previous programmes and possibilities for the future. Biocontrol News and Information, Oxfordshire, v. 11, p. 107-117, 1990.

NJIHIA, T.N.; JARAMILLO, J.; MURUNGI, L.; MWENDA, D.; ORINDI, B.; POEHLING, H.; TORTO, B. Spiroacetals in the colonization behaviour of the coffee berry borer: A ‘push-pull’ system. PLoS ONE, São Francisco, v. 9, n. 11, p. 1-15, 2014.

OLIVEIRA, C.M.; AUAD, A.M.; MENDES, S.M.; FRIZZAS, M.R. Economic impact of exotic insect pests in Brazilian agriculture. Journal of Applied Entomology, Berlin, v. 137, p. 1-15, 2013.

ORTIZ, A.; VEGA, F.E.; POSADA, F.J. Volatile composition of coffee berries at different stages of ripeness and their possible attraction to the coffee berry borer 
Hypothenemus hampei (Coleoptera: Curculionidae). Journal of Agriculture and Food Chemistry, Easton, v. 52, p. 5914-5918, 2004.

PAINE, T.D.; RAFFA, K.F.; HARRINGTON, T.C. Interactions among scolytid bark beetles, their associated fungi, and live host conifers. Annual Review of Entomology, Palo Alto, v. 42, p.179-206, 1997. 
PEREIRA, A.E.; VILELA, E.F.; TINOCO, R.S.; DE LIMA, J.O.G; FANTINE, A.K.; MORAIS, E.G.F; FRANÇA, C.F.M. Correlation between numbers captured and infestation levels of the Coffee Berry-borer, Hypothenemus hampei: A preliminary basis for an action threshold using baited traps. International Journal of Pest Management, Maryland, v. 58, p. 183-190, 2012.

PERFECTO, I.; VANDERMEER, J. The effect of an ant-hemipteran mutualism on the coffee berry borer (Hypothenemus hampei) in southern Mexico. Agriculture,

Ecosystems and Environment, Amsterdam, v. 117, p. 218-221, 2006.

PERFECTO, I.; RICE, R. A.; GREENBERG, R.; VAN DER VOORT, M. E. Shade Coffee: A Disappearing Refuge for Biodiversity. BioScience, Oxford, v. 46, p. 598-608, 1996.

PHILPOTT, S.M.; ARMBRECHT, I. Biodiversity in tropical agroforests and the ecological role of ants and ant diversity in predatory function. Ecological Entomology, London, v. 31, issue 4, p. 369-377, 2006.

RAFFA, K.F.; BERRYMAN, A.A. interacting selective pressures in conifer-bark beetle systems: a basis for reciprocal adaptations? The American Naturalist, Chicago v. 129, p. 234-262, 1987.

ROBLERO, E.N.C.; MALO, E.A. Chemical analysis of coffee berry volatiles that elicit an antennal response from the coffee berry borer Hypothenemus hampei. Journal of the Mexican Chemical Society, Mexico D. F., v. 57, n. 4, p. 321-327, 2013.

SCHROEDER, L.M.; LINDELOW, A. Attraction of scolytids and associated beetles by different absolute amounts and proportions of $\alpha$-pinene and ethanol). Journal of Chemical Ecology, Dordrecht, v. 15, p. 807-817, 1989.

SHEARER, D.A.; BOCH, R. Citral in the nassanoff pheromone of the honeybee. Insect Physiology, Oxford, v. 12, p. 1513-1521, 1966.

SHEPHERD, W.P.; HUBER, D.P.W.; SEYBOLD, S.J.; FETTIG, C.J. Antennal responses of the western pine beetle, Dendroctonus brevicomis (Coleoptera: Curculionidae), to stem volatiles of its primary host, Pinus ponderosa, and nine sympatric nonhost angiosperms and conifers. Chemoecology, Basel, v. 17, p. 209-221, 2008. 
SHEPHERD, W.P.; SULLIVAN, B.T. Southern Pine Beetle, Dendroctonus frontalis, Antennal and Behavioral Responses to Nonhost Leaf and Bark Volatiles. Journal of Chemical Ecology, Dordrecht, v. 39, p. 481-493, 2013.

SILVA, W. D. Idade de fêmeas colonizadoras de Hypothenemus hampei (Ferrari) e sua resposta aos voláteis de flores de café, Coffea arabica L. - 2014. 56p. Tese eCiências com ênfase em Entomologia) - Escola Superior de Agricultura "Luiz de Queiroz”, Universidade de São Paulo, Piracicaba, São Paulo, Brasil, 2014.

SILVA, W.D.; MASCARIN, G.M.; ROMAGNOLI, E.M; BENTO, J.M.S. Mating Behavior of the Coffee Berry Borer, Hypothenemus hampei (Ferrari) (Coleoptera: Curculionidae: Scolytinae). Journal of Insect Behavior, New York, v. 25, p. 408-417, 2012.

SILVA, F.C. da; VENTURA, M.U.; MORALES, L. Capture of Hypothenemus hampei Ferrari (Coleoptera, Scolytidae) in response to trap characteristics. Scientia Agrícola, Piracicaba, v. 63, p. 567-571, 2006.

SOKAL, R.R.; ROHLF, F.J. Biometry.3rd ed. New York: WH Freeman, 1995.

STASHENKO, E.E.; MARTÍNEZ, J.R.; CÁRDENAS-VARGAS, S.; SAAVEDRABARRERA, R.; DURÁN. D. C. GC-MS study of compounds isolated from Coffea arabica flowers by different extraction techniques. Journal of Separation Science, Weinhein, v. 36, p. 2901-2914, 2013.

SZENDREI, Z.; MALO, E.; STELINSKI, L.; RODRIGUEZ-SAONA, C. Response of Cranberry Weevil (Coleoptera: Curculionidae) to Host Plant Volatiles. Environmental Entomology, Lanham, v. 38, p. 861-869, 2009.

TICHELER, J.H.G. Estudio analítico de la epidemiologia del escolítido de los granos de café Stephanoderes hampei Ferr. en Costa de Marfil. Cenicafé, Chinchina, v. 14, p. 223-294, 1963.

VARÓN, E.H.; HANSON, P.; BORBÓN, O.; CARBALLO, M.; HILJE, L. Potencial de hormigas como depredadores de la broca del café (Hypothenemus hampei) em Costa Rica. Manejo Integrado de Plagas y Agroecologia, Turrialba, v. 73, p. 42-50, 2004.

VEGA, F.E. Coffee berry borer, Hypothenemus hampei (Ferrari) (Coleoptera: Curculionidae: Scolytinae). 2nd ed. -In: CAPINERA, J.L. (Ed.). Encyclopedia of Entomology, New York: Springer, 2008, p. 959-960. 
VEGA, F.E.; BENAVIDES, P.; STUART, J.A.; O’NEILL, S.L. Wolbachia Infection in the Coffee Berry Borer (Coleoptera: Scolytidae). Annals of the Entomological Society of America, College Park, v. 95, n. 3, p. 374-378, 2002.

VEGA, F.E.; DAVIS, A. P.; JARAMILLO, J. From forest to plantation? Obscure articles reveal alternative host plants for coffee berry borer, Hypothenemus hampei. Biological Journey of Linnean Society, London, v. 107, p. 86-94, 2012.

VEGA, F.E.; INFANTE, F.; CASTILLO, A.; JARAMILLO, J. The coffee berry borer, Hypothenemus hampei (Ferrari) (Coleoptera: Curculionidae): a short review, with recent findings and future research directions. Terrestrial Arthropod Reviews, Washington, v. 2, p. 129-147, 2009.

VEGA, F.E.; KRAMER, M.; JARAMILLO, J. Increasing coffee berry borer (Coleoptera: Curculionidae: Scolytinae) female density in artificial diet decreases fecundity. Journal of Economic Entomology, Lanham, v. 104, p. 87-93, 2011.

VELASCO PASCUAL, H.; LLAVÉN-GÓMEZ, J.M.; VELÁSQUEZ-VELÁSQUEZ, A.F. Respuesta a extractos de cerezas de café utilizados como atrayente para hembras intercosecha de la broca del fruto Hypothenemus hampei Ferr. In: SIMPOSIO LATINOAMERICANO DE CAFICULTURA, 18., 1997, San José. Memorias..., San José, 1997, p. 349-352.

VÉLEZ, M. Hormigas y su papel en el control biológico de la broca del café. In: Memorias Curso Internacional Teórico - Práctico. Sección II. Parasitoides y otros enemigos de la broca del café. Cenicafé, Chinchiná, marzo 18 al 22, 2002, p. 15-23.

VILLACORTA, A.; POSSAGNOLO, A.F.; SILVA, R.Z; RODRIGUES, P. S. Um modelo de armadilha com semioquímicos para o manejo integrado da broca do café Hypothenemus hampei (Ferrari) no Paraná. In; SIMPÓSIO DE PESQUISA DOS CAFÉS DO BRASIL,2., 2001, Vitória. Anais... Vitória, 2001. p. 2093-2098.

VILLANUEVA, E. Efectividad del control manual y químico para la broca del café, $H$. hampei en el Soconusco, Chiapas, México. In: SIMPÓSIO Taller Regional sobre la Broca del Fruto del Cafeto, 1990, San Salvador. (4), IICA-Promecafé, San Salvador, El Salvador, 1990, 3p.

WEGBE, K.; CILAS, C.; DECAZY, B.; ALAUZET, C.; DUFOUR, B. Estimation of production losses caused by the coffee berry borer (Coleoptera: Scolytidae) and calculation of an economic damage threshold in Togolese coffee plots. Journal of Economic Entomology, Lanham, v. 96, p. 1473-1478, 2003. 
WOOD, D.L. The role of pheromones, kairomones and allomones in the host selection and colonization of bark beetles. Annual Review of Entomology, Palo Alto, v. 27, p. 411-446, 1982.

WRIGLEY, G. Coffee. Longman Scientific \&Technical. Essex: Cambridge, 1988. 639p.

ZHANG, Q.H.; BIRGERSSON, G.; ZHU, J.W.; LOFSTEDT, C.; LOFQVIST, J.;

SCHLYTER, F.. Leaf volatiles from nonhost deciduous trees: Variation by tree species, season and temperature, and electrophysiological activity in Ips typographus. Journal of Chemical Ecology, Dordrecht, v. 25, p. 1923-1943, 1999. 